\title{
Evaluación del estado nutricional del adulto mayor en una estancia geriátrica permanente
}

MTFS Claudia Marcela Cantú Sánchez. cm.cantusanchez@ugto.mx Profesor de Tiempo Completo. Doctorante en Desarrollo Humano, Departamento de Enfermería y Obstetricia. División de Ciencias de la vida, México. Línea de Investigación Promoción de la salud y cuidado en grupos vulnerables.

Dra. Gloria Vega Argote. gloriav@ugto.mx

Profesor de Tiempo Completo. Departamento de Enfermería y Obstetricia de Irapuato.

División de Ciencias de la Vida. Universidad de Guanajuato. México. Línea de Investigación Prevención y estados crónicos del adulto.

ME. Greever María Ávila Sánsores. greavila@ugto.mx Profesor de Tiempo Completo. Departamento de Enfermería y Obstetricia de Irapuato. División de Ciencias de la Vida. Universidad de Guanajuato. México. Línea de Investigación Prevención y estados crónicos del adulto.

Dr. Osmar Antonio Jaramillo Morales oa.jaramillo@ugto.mx Profesor de Tiempo Completo. Departamento de Enfermería y Obstetricia de Irapuato.

División de Ciencias de la Vida. Universidad de Guanajuato, México Línea de Investigación Promoción de la salud y cuidado en grupos vulnerables

\section{RESUMEN}

El proceso de envejecimiento sugiere una serie de cambios fisiológicos a nivel de los diversos órganos y sistemas provocando pérdida de la masa y fuerza conocida como sarcopenia. La desnutrición es uno de los grandes síndromes geriátricos y un factor desencadenante para fragilidad en los adultos mayores. Esto representa un factor de 
riesgo para institucionalización y desencadenamiento de otras morbilidades. Es por esto que la realización de un diagnóstico oportuno sobre el estado nutricional de los adultos mayores dentro de una estancia geriátrica permanente es una herramienta para la realización y evaluación de las intervenciones de enfermería para los cuidadores y los pacientes institucionalizados. El presente trabajo fue parte de la materia de Cuidado de Enfermería en la salud mental donde los estudiantes de la materia de Cuidado de Enfermería en el adulto mayor tomaron los datos a evaluar a través de 4 escalas de evaluación que fueron la escala de Braden para determinar úlceras por presión, Escala de Morse para evaluar riesgo de caídas, Mini nutricional Self MNA para evaluar estado nutricional y SARC - F que evalúa presencia de sarcopenia. Dentro de los principales resultados encontramos que los adultos mayores institucionalizados presentan desnutrición $17 \%$, 30\% riesgo de desnutrición, $45 \%$ estado nutricional normal y un $8 \%$ se negó a evaluar, en cuanto a riesgo bajo de úlceras por presión, con un poco riesgo de caídas con un $50 \%$ y 25 sin riesgo de caídas, en cuanto a la presencia de úlceras se encontró un $70 \%$ de riesgo bajo, $15 \%$ con riesgo moderado y $15 \%$ de pacientes con alto riesgo de presentar úlceras por presión. Es por esto que la evaluación y seguimiento de la nutrición tanto de cuidadores como de personal de enfermería son cruciales para evitar compromiso en la salud del adulto mayor.

Palabras clase: adulto mayor; desnutrición; estancia geriátrica. 


\title{
Evaluation of the nutritional status of the elderly in \\ a permanent geriatric stay
}

\begin{abstract}
The aging process suggests a series of physiological changes at the level of various organs and systems, causing loss of mass and strength known as sarcopenia. Malnutrition is one of the major geriatric syndromes and a triggering factor for frailty in older adults. This represents a risk factor for institutionalization and triggering of other morbidities. This is why making a timely diagnosis of the nutritional status of older adults within a permanent geriatric stay is a tool for carrying out and evaluating nursing interventions for caregivers and institutionalized patients. The present work was part of the subject of Nursing Care in mental health where the students of the subject of Nursing Care in the elderly took the data to be evaluated through 4 evaluation scales that were the Braden scale to determine pressure ulcers, Morse Scale to assess the risk of falls, Mini nutritional Self MNA to assess nutritional status and SARC - F that assesses the presence of sarcopenia. Among the main results we found that institutionalized older adults have $17 \%$ malnutrition, $30 \%$ risk of malnutrition, $45 \%$ normal nutritional status and $8 \%$ refused to evaluate, in terms of low risk of pressure ulcers, with a little risk of falls with $50 \%$ and 25 without risk of falls, in terms of the presence of ulcers, $70 \%$ were found to be at low risk, $15 \%$ at moderate risk and $15 \%$ of patients at high risk of presenting pressure ulcers. This is why the evaluation and follow-up of the nutrition of both caregivers and nursing staff are crucial to avoid compromising the health of the elderly.
\end{abstract}

Keywords: older adult; malnutrition; geriatric stay.

Artículo recibido: 02 enero 2022 Aceptado para publicación: 28 enero 2022 Correspondencia: cm.cantusanchez@ugto.mx Conflictos de Interés: Ninguna que declarar 


\section{INTRODUCCIÓN}

La vejez es la etapa de la vida cuyo inicio es determinado por cada sociedad. Actualmente, en los países en desarrollo como México se acepta como inicio de la vejez los 60 años, mientras que en los países desarrollados esa edad es a los 65 años. Cuando nos referimos a envejecimiento, hablamos de un proceso natural, gradual, continuo e irreversible de cambios a través del tiempo. Estos cambios se dan en el nivel biológico, psicológico y social, que están determinados por la historia, la cultura y las condiciones socioeconómicas de los grupos y las personas. Por ello, la forma de envejecer de cada persona es diferente. Si bien, el envejecimiento debería ser sano y activo, existen problemas de salud en los adultos mayores como grupo poblacional en este caso las enfermedades crónicas, que tienen mayor prevalencia y ocurren por múltiples factores, es por esto que la familia y sociedad tienen la responsabilidad de ofrecer las oportunidades para que el adulto mayor tenga las habilidades para lograr un autocuidado satisfactorio. De acuerdo con la pirámide poblacional en el estado de Guanajuato se visualizó un aumento en el grupo de Adultos Mayores del 6.5\% en el año 2000 y del $13.9 \%$ en el año 2015, y donde será más notable por su rapidez será en el corredor Industrial que incluye a la ciudad de León, Irapuato y Celaya. Los problemas de salud que se presentan con más frecuencia son: diabetes, cáncer, neumonía, depresión, sordera, ceguera, enfermedades no identificadas por proceso de envejecimiento.

En el año actual 2021, se observa un aumento de $7.83 \%$ en la cantidad de adultos mayores, es por esto que se debe contar con la creación de empresas al cuidado de ellos, y con esto un crecimiento en las áreas de enfermería, optometría, odontología y especialidades médicas enfocadas en el adulto mayor. Como se mencionó anteriormente en el envejecimiento la persona atraviesa por cambios biopsicosociales importantes que pueden hacer que la dinámica cotidiana del adulto se altere, también aumenta la prevalencia de problemas ligados al estado nutricional, que van desde la desnutrición hasta el sobrepeso y la obesidad. Por tal razón, la nutrición vista como el factor más influyente en la salud de los adultos mayores debe ser un aspecto vital a tener en cuenta en la intervención por parte del personal de enfermería, desde la promoción de la salud y la prevención de la enfermedad que tienen su base en la modificación de hábitos y conductas para incorporar otras como: la realización de actividad física regular, disminución del consumo de alcohol o tabaco y la adopción de una dieta saludable 
adaptada a las necesidades propias del envejecimiento. Estas acciones deben ser parte de un tratamiento conjunto en el mantenimiento integral de la salud y control de las enfermedades crónicas, para proporcionar así una mejor calidad de vida al individuo en donde se pueda adaptar a su nueva condición.

\section{Justificación}

Se torna indispensable una buena alimentación, para aminorar el deterioro progresivo en la fisiología del adulto mayor y la prevención de las enfermedades que afectan en gran parte a este grupo etéreo (sarcopenia, artritis, artrosis, osteoporosis, diabetes, hipertensión arterial, infartos cardiacos).

La importancia en realizar una valoración del estado nutricional reside en obtener datos sobre la calidad de la salud de los adultos mayores, encontrar algún problema ligado a éste o riesgo de desnutrición, la obtención de otros diagnósticos y el desarrollo de un plan para intervenir y dar seguimiento.

Se pretende que la investigación aporte conocimientos sobre la situación nutricional del adulto mayor que reside en "Casa Hogar La Paz" y la relación que esta tiene con la sarcopenia, para que con la participación de las personas que le rodeen, se logre mejorar su calidad de vida.

\section{Adulto Mayor}

Se denomina envejecimiento al proceso de cambios morfofuncionales que se producen en el ser vivo en relación con el paso del tiempo. Dicho proceso de envejecimiento puede ser contemplado desde dos perspectivas principales, la referida a los cambios fisiológicos o esperados para todos los individuos independientemente de sus experiencias vitales, o lo que se refiere a los cambios patológicos, propios de cada individuo y que están relacionados con las alteraciones del equilibrio orgánico en relación con el desarrollo de procesos morbosos. En el año de 1987, Rowe y Kahn establecieron la diferenciación entre envejecimiento normal (usual aging) y envejecimiento exitoso o saludable (succesful aging), incluyendo en éste la baja probabilidad de padecer enfermedades o discapacidad, elevada capacidad funcional física y cognitiva, y mantenimiento de una vida activa en la sociedad.

Se tiene estimado que únicamente entre el 20 y $25 \%$ de la variabilidad en la edad de fallecimiento vendrá determinado por los factores genéticos, de los cuales unos se constituirán en factores de supervivencia, mientras que otros serán factores 
predisponentes para el padecimiento de ciertas enfermedades crónicas, entre las que podríamos destacar las enfermedades coronarias, algunos tipos de cáncer, la diabetes mellitus o algunas demencias como la enfermedad de Alzheimer.

Los determinantes socioeconómicos ejercen una gran influencia sobre el proceso de envejecimiento, de tal manera que en las sociedades desarrolladas y, por lo tanto, más ricas, la esperanza de vida al nacer llega a ser el doble de la de los países más pobres, incluyéndose las condiciones y seguridad en el trabajo, aspectos referidos a la jubilación o el nivel de instrucción alcanzado.

Otro factor de gran relevancia en el proceso de envejecimiento como lo refiere Calenti, 2011, será el referido a los hábitos o estilos de vida; así, se considerarían hábitos anómalos y, por lo tanto, con influencia negativa sobre el envejecimiento, la vida sedentaria, el consumo de tabaco o alcohol, las dietas no equilibradas, la falta de las relaciones sociales o la actividad en algunos sectores laborales generadores de estrés (directivos, profesionales de la salud o controladores aéreos, entre otros), o muy exigentes desde el punto de vista físico (estibadores o peones de obra, entre otros). Hay que señalar la importante diferencia de longevidad en cuanto al género, ya que, en las mujeres, por ejemplo, en España, viven casi siete años más de media que los hombres y, de alguna manera, están condicionando una sociedad feminizada de mayores, sobre todos cuando nos referimos a la población de más edad.

El envejecimiento es el conjunto de transformaciones y/o cambios que aparecen en el individuo a lo largo de la vida; es la consecuencia de la acción del tiempo sobre los seres vivos. Estos cambios son: bioquímicos, fisiológicos, morfológicos, sociales, psicológicos y funcionales. (Sociedad Española de Enfermería Geriátrica, 1999)

\section{La edad efectiva de una persona puede establecerse teniendo en cuenta diversas consideraciones que nos permiten diferencias cuatro tipos de edades: Edad cronológica, Edad fisiológica, Edad psíquica y Edad social.}

La Edad Cronológica: Se define por haber cumplido un determinado número de años; respecto de la vejez se ha convenido hasta el momento, los 65 años. Es objetiva en su medida ya que todas las personas nacidas en la misma fecha comparten idéntica edad cronológica. La ventaja de la objetividad de la edad cronológica se vuelve inconveniente al comprobar el impacto diferente del tiempo sino la calidad del tiempo transcurrido, los acontecimientos vividos y las condiciones ambientales que lo han rodeado. 
Edad Fisiológica: Es definida por el envejecimiento de sus órganos y tejidos, es decir, la afectación física del individuo. Los cambios orgánicos se producen de forma gradual: lentos e inapreciables al comienzo del proceso, hasta que afectan el normal desarrollo de las actividades de la vida diaria o interfieren en ellas.

Edad Psíquica: Difícilmente se puede establecer, pero los acontecimientos externos de la vida de cada persona, sociales y afectivos, hacen reaccionar a cada una según su personalidad, circunstancias y experiencia vital.

Edad Social: Esta se establece con el rol individual que se debe desempeñar en la sociedad. Esta forma de clasificar puede considerarse discriminatoria ya que no tiene en cuenta las aptitudes y actitudes personales frente a la resolución de las actividades de la vida diaria. Sin embargo, los límites de la edad social cambian según las necesidades económicas y políticas del momento. (Sociedad Española de Enfermería Geriátrica, 1999).

\section{El Paciente Gerontológico como persona: autonomía y dependencia.}

Nuestras sociedades envejecidas plantean desafíos económicos, sociales, familiares, personales y también éticos. El tránsito a la madurez y el logro de autonomía significan convertirse en razonadores prácticos independientes. No independencia en el sentido de autarquía, sino independencia consciente de nuestra vulnerabilidad, fragilidad y dependencia: una interdependencia reflexiva, en la que el reconocimiento de la dependencia y su conversión en oportunidad o capacidad de vida es la clave de la independencia y la autonomía.

\section{Las tres dimensiones de la autonomía}

Para identificar y comprender cuáles son las necesidades de salud de un paciente gerontológico y las respuestas clínicas y éticas más adecuadas es necesario distinguir tres dimensiones de la autonomía, aunque no como realidades aisladas sino como un continuum: la autonomía decisoria, la autonomía informativa y la autonomía funcional:

La autonomía decisoria refiere a la libertad de elección, esto es, a la capacidad del paciente para deliberar y decidir (se) por un curso de acción. Es la dimensión ética y jurídicamente más desarrollada, a partir de la teoría del consentimiento informado. La ausencia o limitación de la autonomía decisoria se califica genéricamente como incapacidad y se soluciona a través de las decisiones de representación, las instituciones 
de guarda, representación y asistencia y más recientemente, la planificación anticipada de la atención o las instrucciones previas.

La autonomía informativa se centra en la definición, la protección y el control de la información de carácter personal y de la documentación asistencial, bien con mecanismos tradicionales como el secreto profesional, la confidencialidad y el derecho a la intimidad, bien con otros más recientes como el derecho fundamental a la protección de datos personales, garantizados al paciente anciano igual que al resto de los ciudadanos.

La autonomía funcional alude a la libertad de acción (actuación o abstención) y la capacidad de realizar por uno mismo las decisiones adoptadas. Esta dimensión está estrechamente relacionada con la primera, la autonomía decisoria, pero conviene otorgarle un tratamiento individualizado en el caso del paciente gerontológico. Las limitaciones en la autonomía funcional suelen calificarse como situaciones de dependencia, o discapacidad y su solución no proviene de las instituciones de representación (que en ocasiones son innecesarias e ineficaces), sino de otras como la asistencia personal, los sistemas de apoyo y los cuidados, profesionales y no profesionales o informales. (Calenti, 2011).

\section{Análisis de la actividad}

Diversas investigaciones centradas en el grupo de población de personas mayores concluyen que la actividad física, psicológica y social aumenta de forma significativa la calidad de vida y el bienestar en las personas que lo realizan de manera asidua. Los individuos experimentan por sí mismos y es a raíz de estas experiencias donde conocen sus capacidades y déficit. Así pues, el profesional de la salud puede utilizar las actividades para evaluar las destrezas y déficit de cada persona y para identificar las subsecuentes razones para la planificación de la intervención terapéutica basada en diversas actividades.

\section{Desempeño Ocupacional}

El objetivo de la terapia ocupacional es convertir estas ocupaciones humanas que son habituales en estado de salud (comer, caminar, etc) en actividades que adquieren circunstancialmente valor terapéutico.

Para explicar adecuadamente qué es el desempeño ocupacional es necesario entender que desde la terapia ocupacional se trata la función y son utilizados los procedimientos y actividades con el propósito de promover la salud y el bienestar ( por ejemplo, a través 
de la higiene postural); minimizar o prevenir el deterioro (por ejemplo, a través de estimulación cognitiva con respecto a la memoria); desarrollar, mantener, mejorar y/o recuperar el desempeño de las funciones necesarias y, por último, desarrollar, mantener y compensar las disfunciones instauradas. Las categorías de la función se encuentran bien diferenciadas: por un lado, las áreas del desempeño ocupacional y, por otro, los componentes del desempeño ocupacional. Según el Marco de Trabajo de la Práctica de la Terapia Ocupacional en su segunda edición (2008) las áreas del desempeño son:

Las actividades de la Vida Diaria Básicas son aquellas actividades orientadas al cuidado de uno mismo, e incluyen el nivel más básico de la ocupación. Incluye la alimentación, el vestido, higiene menor (lavado de manos, lavado de cara, cepillado de pelo y cepillado de dientes), higiene mayor (uso del baño), esfínteres, movilidad funcional (en la cama, silla de ruedas y transferencias) y cuidado de dispositivos personales (gafas, audífonos, lentillas, dentadura postiza, equipamiento adaptado y dispositivos sexuales $\mathrm{y}$ contraceptivos).

Las actividades de la Vida Diaria Instrumentales son aquellas que están orientadas hacia la interacción con el medio y que son a menudo más complejas. Incluye cuidado de otros, cuidado de mascotas, uso de sistemas de comunicación (teléfono e internet), uso de transporte público, manejo de dinero, cuidados del hogar, preparación de comidas, medidas de mantenimiento y seguridad en el hogar e ir de compras.

\section{Percepción de los cuidados al adulto mayor}

Con la globalización debemos retomar el concepto de "cuidar es una actividad humana que se define como una relación y un proceso cuyo objetivo va más allá de la enfermedad (Colliere, 2007) debido al aumento en la esperanza de vida a la disminución de la tasa de natalidad, la población de adultos mayores está creciendo más rápido en comparación con otro grupo de edad, lo que indica que la población mundial está envejeciendo a pasos acelerados, pero eso también habla de que existen programas exitosos en salud pública y desarrollo socioeconómico haciendo a los adultos mayores funcionales, se prevé que la mayoría de los adultos mayores tendrán convivencia con alguno de sus padres vivos, y muchos jóvenes conocerán a sus bisabuelos, esto ya es cotidiano en nuestros tiempos. La percepción hacia la persona adulta mayor ha ido variando, antes era importante que estuviera inmerso en la familia, más sin embargo ahora los familiares ya consideran el ingreso de ellos en centros geriátricos. Una de las propuestas que los cuidados que se 
realizan en un centro residencial se realicen por el personal de enfermería ya que se basan en la planeación asistida de personas ancianos que no pueden permanecer al cuidado de familiares en su propio domicilio, estos deben cumplir con criterios de calidad en la estructura y funcionamiento, sobre todo porque este será su hogar y debe brindar seguridad sobre todo para la prevención de caídas. El personal de enfermería por su formación es el único que debe de estar capacitado para la realización de determinadas técnicas como la administración de medicamentos, aplicación de sondas nasogástricas, valoración biopsicosocial para que el equipo interdisciplinario realice las medidas terapéuticas pertinentes de acuerdo con las necesidades del paciente. Pero además encontramos situaciones en emergencia como Accidentes cerebrovasculares, caídas, glaucoma, asfixia, por lo que se requiere una valoración integral para realizar intervenciones inmediatas de manera adecuada, debemos tomar en cuenta también los factores de riesgo para aplicar programas preventivos, de tratamiento y rehabilitación. (Quintanilla, 2006).

Otro dato importante a considerar es que los adultos mayores que necesitan de cuidados se deben sentir confiados y seguros si conocen a las personas que les brindan cuidados, aplicando los conocimientos en el manejo de Técnicas y procedimientos ya que su seguridad es muy importante, ya que si el cuidado se realiza de forma no satisfactoria, tardarán más en llegar al bienestar, además los apoyos psíquicos y emocionales tienen un gran impacto en su salud mental fortaleciendo la experiencia como parte del crecimiento personal. (Bergland, 2006). Rey J.I. citado en Vanegas 2009, refiere que el aumento de los adultos mayores lo coloca en una posición de vulnerabilidad en su salud, y protección por lo que debe aplicarse sus derechos humanos y del adulto mayor en las instituciones geriátricas con profesionales disponibles resaltando la importancia del personal de enfermería profesional y capacitado para brindar atención en el cuidado de la salud, seguridad, alimentación, abrigo y trato digno, ya que el objetivo de los centros geriátricos es lograr la mayor autonomía y la valía en actividades de la vida diaria.

Ante estos datos del crecimiento de la población de adultos mayores, Hidalgo 2012 espera que realicen acciones encaminadas a un autocuidado, haciendo referencia a Dorothea Orem y su teoría del autocuidado que se define como el cuidado de uno mismo, dado por uno mismo y para uno mismo, que es una función inherente a los profesionales de la salud involucrando a la familia y su entorno, incorporándolo a actividades de la vida 
diaria para aumentar la calidad de vida, es una actividad aprendida por los individuos para lograr un beneficio de su vida, salud o bienestar. Es un derecho del adulto mayor que participe en los programas de salud público y privados, para lograr el autocuidado a través de la consecución de objetivos, ya que al mejorar los conocimientos sobre aspectos de salud, factores de riesgo y promoción de la salud, además si la persona es sensible a sus sentimientos, sensaciones corporales y respuestas valorara las necesidades personales y aumentara la capacidad de auto cuidarse y lograremos tratar con una población adulta más saludable.(Orem, 1995). La capacidad de realizar el autocuidado aumenta la independencia percibida por las personas mayores aumentando la independencia aumenta la autoestima, la autoconfianza aumentando el bienestar emocional de las personas, la familia es un factor importante para que se realice el autocuidado, así como el personal sanitario a cargo. (Hidalgo, 2012).

\section{Objetivo General}

- Con este diagnóstico situacional buscamos recabar información específica y fidedigna por medio de escalas de medición acerca del estado nutricional en el que se encuentran los integrantes de la casa hogar "La Paz", esto con el fin de determinar medidas preventivas o, en todo caso, correctivas que se vean involucradas en la alimentación, riesgo de desnutrición y sarcopenia de los pacientes.

\section{Objetivos Específicos}

- Realizar el llenado de escala Mini Nutritional Asessment contestándola de manera eficaz por cada adulto mayor designado a cada colaborador para designar intervenciones de enfermería registrando la efectividad de cada caso.

- Determinar estado de la nutrición del adulto mayor

\section{Marco de referencia}

Concepto de Norma Oficial Mexicana y su reglamentación

Las normas oficiales mexicanas (NOM) son disposiciones generales de tipo técnico expedidas por dependencias de la administración pública federal.

Su objetivo es establecer reglas, especificaciones, directrices y características aplicables a un producto, proceso o servicio. 


\section{Objetivo}

Esta Norma Oficial Mexicana tiene por objeto establecer los procedimientos para uniformar principios, criterios, políticas y estrategias en la prestación de servicios y desarrollo de actividades en materia de asistencia social a menores y adultos mayores.

\section{Campo de aplicación}

Esta Norma Oficial Mexicana es aplicable a todas las dependencias y entidades de la administración pública, tanto federal como local y las personas físicas o morales de los sectores social y privado que conforman el Sistema Nacional de Salud.

- Adulto mayor, a la persona de 60 años de edad o más.

- Adulto mayor en estado de abandono, aquel que presenta cualquiera de las características siguientes: carencia de familia, rechazo familiar, maltrato físico o mental, carencia de recursos económicos.

- Albergue para adultos mayores, al establecimiento donde se proporcionan temporalmente servicios de alojamiento a adultos mayores, en tanto son reubicados a otras instituciones o a su familia.

- Casa hogar para adultos mayores, al establecimiento de asistencia social donde se proporciona a adultos mayores atención integral mediante servicios de alojamiento, alimentación, vestido, atención médica, trabajo social, actividades culturales, recreativas, ocupacionales y psicológicas

Prestación de servicios de asistencia social en casa hogar y albergues para adultos mayores deben incluir:

\section{Alimentación}

- El usuario tendrá derecho a recibir tres alimentos al día con un intervalo de seis a siete horas entre un alimento y otro.

- La alimentación debe ser de buen sabor y aspecto, en cantidad suficiente para una adecuada nutrición y servida en utensilios decorosos.

\section{Atención médica}

\section{Se debe contar con equipo médico indispensable que incluya:}

a. Botiquín de primeros auxilios.

b. Estetoscopio biauricular.

c. Esfigmomanómetro.

d. Estuche de diagnóstico con oftalmoscopio. 
e. Aspirador.

La atención médica que se proporcione al adulto mayor debe estar sustentada en principios científicos, éticos y sociales; comprende actividades preventivas, curativas y de rehabilitación, que se llevan a cabo por el médico, la enfermera, gericultista, psicólogo y terapista físico - ocupacional

Con sustento a la NORMA OFICIAL MEXICANA NOM-167-SSA1-1997, PARA LA PRESTACION DE SERVICIOS DE ASISTENCIA SOCIAL PARA MENORES Y ADULTOS MAYORES, enfocado en el asilo de ancianos "Casa Hogar la Paz" y al Diagnostico nutricional encontramos:

\subsection{Alimentación.}

8.2.1 El usuario tendrá derecho a recibir tres alimentos al día con un intervalo de seis a siete horas entre un alimento y otro.

8.2.2 La alimentación debe ser de buen sabor y aspecto, en cantidad suficiente para una adecuada nutrición y servida en utensilios decorosos. 8.3 Atención médica.

8.3.1 Se debe contar con equipo médico indispensable que incluya: a) Botiquín de primeros auxilios. b) Estetoscopio biauricular. c) Esfigmomanómetro. d) Estuche de diagnóstico con oftalmoscopio. e) Aspirador. 8.3.2 La atención médica que se proporcione al adulto mayor debe estar sustentada en principios científicos, éticos y sociales; comprende actividades preventivas, curativas y de rehabilitación, que se llevan a cabo por el médico, la enfermera, gericultista, psicólogo y terapista físico ocupacional.

8.3.3 Las actividades preventivas comprenderán las acciones siguientes:

8.3.3.1 Educación y promoción de la salud del adulto mayor.

8.3.3.2 Fomento de una cultura de dignificación del adulto mayor, que implica la difusión de preceptos gerontológicos que permitan conocer y comprender el proceso de envejecimiento.

8.3.3.3 Detección oportuna de factores de riesgo y enfermedades, para prevenir secuelas discapacitantes y mantener la funcionalidad y autonomía del individuo, entre las cuales se encuentran: a. Hipertensión arterial. b. Diabetes mellitus. c. Enfermedad del sistema músculo esquelético. d. Aterosclerosis. e. Malnutrición. f. Cáncer. g. Otras enfermedades cardiovasculares. h. Depresión. i. Enfermedades respiratorias. j. Enfermedades visuales. k. Enfermedades auditivas. 1. Enfermedades estomatológicas. 
m. Enfermedades dermatológicas. 8.3.3.4. Diseñar y aplicar programas orientados a la atención integral del adulto mayor con acciones relativas a:

\subsection{Orientación nutricional.}

\subsubsection{Actividades de autocuidado.}

\section{Límites del programa}

El tiempo de elaboración de este programa de forma presencial fue del 22 al 26 de noviembre, por medio de dos turnos en un horario de 9:00 am a 11:00 am y de 11:00 am a 1:00 pm en Casa Hogar de La Paz (asilo de ancianos) ubicada en Avenida de los Insurgentes \#1921, La Paz, Irapuato, Guanajuato, con código postal 36560, donde pudimos conocer y aplicar nuestras intervenciones de enfermería con nuestras personas de cuidado del grupo poblacional de adultos mayores (hombres y mujeres), así como recabar los datos para posteriormente elaborar de forma grupal y virtual en la semana del 29 de noviembre al 3 de diciembre.

\section{MÉTODOS Y METODOLOGÍA}

Se pretende trabajar con pacientes geriátricos que abarcan de 45-90 años que a su vez se dividen en Preseniles 45-60 años, Senectud gradual 60-70 años, Vejez declarada 70-90 años, longevos más de 90 años, que son los pacientes que se encuentran en el albergue del asilo de ancianos Casa hogar la Paz, para determinar el estado nutricional del adulto mayor a través de 4 escalas tipo Likert que son Braden, para ulceras por presión, Riesgo de Caída de Morse, Escala SARC-F para evaluar sarcopenia, y una mini evaluación nutricional, además se contará con una báscula con estadiómetro que ya se encuentra en la institución para determinar el estado nutricional del adulto mayor, se vaciarán los datos en gráficos en Excel para mostrar los datos demográficos y de nutrición.

Actualmente hay un total de 56 adultos mayores institucionalizados en la Casa hogar para ancianos y desvalidos de Irapuato, A.C. se tomaron los criterios de inclusión, pacientes que reunieran los requisitos para ser valorados en su estado nutricional y que aceptaran participar, se excluyó a pacientes con deterioro cognoscitivo y que no aceptaran ser evaluados.

\section{Recursos}

El programa es elaborado por estudiantes de séptimo semestre de la carrera de Enfermería y Obstetricia de la Universidad de Guanajuato, junto a las docentes responsables de la práctica, la MTFS Claudia Marcela Cantú Sánchez como Coordinadora de la práctica la 
MC Greever Ávila Sensores como profesora adjunta, la revisión de la información corrió por parte de la Dra. Gloria Vega Argote y el Dr. Osmar Omar Jaramillo Morales.

Los materiales utilizados de papelería fueron: hojas blancas y tinta donde se imprimió la valoración geriátrica asignada y las escalas de valoración fue financiado por la Empresa Semillas Hibridas de Irapuato, plumas y lápices que los estudiantes llevaron. También se hizo uso de báscula y cinta métrica para medir peso, talla y la pantorrilla. Los medios digitales: computadora donde se trabajó mediante un archivo compartido y la aplicación "Teams" e impresora y en esta ocasión por la pandemia COVID se requirió equipo de protección personal como cubrebocas tricapa, careta, gel antibacterial y sanitizante. Los colaboradores asistieron con uniforme comunitario, de acuerdo con los lineamientos establecidos por la coordinación escolar.

\section{Descripción de las escalas utilizadas}

\section{Escala de Morse}

La escala de caída de Morse es una herramienta rápida y simple para evaluar la probabilidad de que un paciente sufra una caída. Los puntajes se asignan de acuerdo con las siguientes definiciones:

\section{Antecedentes de caídas recientes:}

Se asignan 25 puntos si el paciente se ha caído durante la presente internación o si tiene antecedentes dentro de los últimos tres meses de caídas fisiológicas a consecuencia de situaciones tales como convulsiones o trastornos de la marcha. Si el paciente no se ha caído, se asigna 0 puntos.

Nota: si un paciente se cae por primera vez, entonces su puntaje automáticamente sube a 25. Si el paciente sólo puede caminar apoyándose en los muebles se asignan 30 puntos.

\section{Diagnóstico secundario:}

Se asignan 15 puntos si hay más de un diagnóstico en la historia clínica. Si no, se califica 0 .

\section{Ayuda para deambular:}

Se califica con 0 si el paciente camina sin ningún dispositivo de ayuda (aun siendo ayudado por una enfermera/o), se encuentra en silla de ruedas o está en reposo y no se levanta de la cama para nada. Si el paciente utiliza muletas, bastón o andador se asignan 15 puntos. Si el paciente sólo camina apoyándose en los muebles se asignan 30 puntos. 


\section{Vía endovenosa:}

Se asignan 20 puntos si el paciente tiene una vía IV, en caso contrario el puntaje es 0.

\section{Marcha (Equilibrio y/o traslado):}

Se define como marcha normal cuando el paciente camina con la cabeza erecta, los brazos balanceándose libremente a los costados y con pasos seguros. A esta marcha no se le asignan puntos $(0)$.

Con una marcha débil (puntaje 10) el paciente camina encorvado, pero es capaz de levantar la cabeza mientras camina sin perder equilibrio. Los pasos son cortos y puede arrastrar los pies. Con una marcha alterada (puntaje 20) el paciente puede tener dificultades para levantarse de la silla, pudiendo realizar varios intentos apoyando sus brazos en los brazos de la silla o "tomando IMPULSO" (ej: realizando varios intentos por incorporarse. La cabeza del paciente está baja, mirando al piso. Como tiene muy poco equilibrio, el paciente se agarra de los muebles, de una persona de apoyo o de bastones/andadores y no puede caminar sin esta asistencia).

\section{Conciencia - estado mental:}

Cuando se utiliza esta escala, el estado mental del paciente es valorado chequeando la propia evaluación que hace el paciente acerca de su capacidad para caminar. Se le pregunta al paciente: "¿Puede ir al baño sólo o necesita ayuda?" Si la respuesta del paciente es consistente con sus reales posibilidades se le asigna 0 punto. Si la respuesta del paciente no es realista, se considera que el mismo sobreestima sus propias capacidades y no es consciente de sus limitaciones, asignándose entonces 15 puntos.

\section{Puntuación final y nivel de riesgo:}

Se suman los puntajes de los 6 ítems y se documenta en la historia clínica. Se identifica así el nivel de riesgo de caídas y las acciones recomendadas según el riesgo.

\section{Escala de Braden}

La Escala de Braden fue desarrollada en 1985 en EE.UU., en el contexto de un proyecto de investigación en centros sociosanitarios, como intento de dar respuesta a algunas de las limitaciones de la Escala de Norton. Barbara Braden y Nancy Bergstrom desarrollaron su escala a través de un esquema conceptual en el que reseñaron, ordenaron y relacionaron los conocimientos existentes sobre UPP. 
Escala de Braden evalúa el riesgo de desarrollar úlceras por presión en base a la sensibilidad y humedad de la piel, la fricción del cuerpo con las sábanas, la movilidad, actividad y nutrición del paciente. Un puntaje menor o igual a 16 implica riesgo de desarrollar úlceras por presión, se utilizó este punto de corte ya que es lo que se describe por los autores de la escala para su utilización.

La Escala de Braden consta de seis subescalas: percepción sensorial, exposición de la piel a la humedad, actividad física, movilidad, nutrición, roce y peligro de lesiones cutáneas, con una definición exacta de lo que se debe interpretar en cada uno de los apartados de estos subíndices. Los tres primeros subíndices miden factores relacionados con la exposición a la presión intensa y prolongada, mientras que los otros tres están en relación con la tolerancia de los tejidos a la misma.

\section{Mini Nutricional}

El MNA es una herramienta de cribado que ayuda a identificar a ancianos desnutridos o en riesgo de desnutrición fue validado específicamente en población geriátrica en Toulouse y publicado por primera vez en 1994; desarrollado por Nestlé y geriatras de liderazgo internacional; suficientemente validado en estudios internacionales en varios escenarios clínicos.

Se recomienda repetir el MNA con una periodicidad anual en pacientes ambulatorios, cada tres meses en pacientes institucionalizados o en aquellos que han sido identificados como desnutridos o en riesgo de malnutrición, y cuando ocurra cualquier cambio en la situación clínica del paciente. (Salvà Casanovas, 2012) (NéstleNutritionInstitute.)

\section{Escala de Sarc-F}

SARC-F permite evaluar la fuerza muscular a través de un sistema de evaluación y puntuación en el que los pacientes registran su habilidad en 5 parámetros: fuerza, capacidad para caminar, levantarse de una silla, subir unas escaleras y frecuencia de caídas.

Para cada componente, los pacientes son evaluados con 0,1 , o 2 puntos ( 0 representa ninguna dificultad, 1 supone alguna dificultad y 2 mucha dificultad o incapacidad). La puntuación total va de 0 a 10 y los pacientes que registran 4 puntos o menos padecen sarcopenia. La herramienta SARC-F se ha desarrollado como alternativa a las pruebas de imagen y los test físicos que se utilizan habitualmente para medir la fuerza muscular. 
La sarcopenia es una enfermedad muscular que se caracteriza por una masa muscular baja y una fuerza muscular reducida, como principales determinantes. Si bien es una enfermedad asociada con los adultos mayores, su desarrollo comienza a edades tempranas. Este desorden musculoesquelético progresivo y generalizado está asociado a un incremento del riesgo de caídas, fracturas, discapacidad física y mortalidad. Además del proceso natural de envejecimiento, otros factores, como una alimentación subóptima, la inactividad física, el sedentarismo o el reposo en cama, las enfermedades crónicas y/o determinados tratamientos farmacológicos, pueden favorecer el desarrollo de sarcopenia.

\section{RESULTADOS}

Valoración Self-MNA 2012, Mini Nutritional Assessment, para adultos a partir de 65 años.

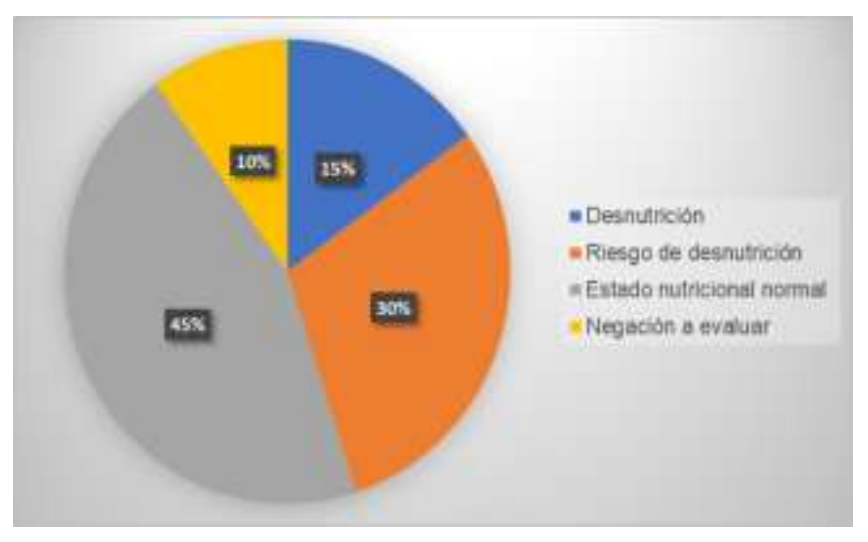

La escala Self-MNA 2012, se aplicó a 20 pacientes, arrojando los siguientes resultados: 3 pacientes $(17 \%)$ con desnutrición, 6 pacientes $(30 \%)$ con riesgo de desnutrición, 9 pacientes $(45 \%)$ con un estado nutricional normal y 2 pacientes de negaron a evaluar. Valoración Escala SARC-F.

La escala SARC-F, se aplicó a 20 pacientes, arrojando los siguientes resultados: 13 pacientes $(65 \%)$ con sarcopenia y 7 pacientes $(35 \%)$ sin sarcopenia.

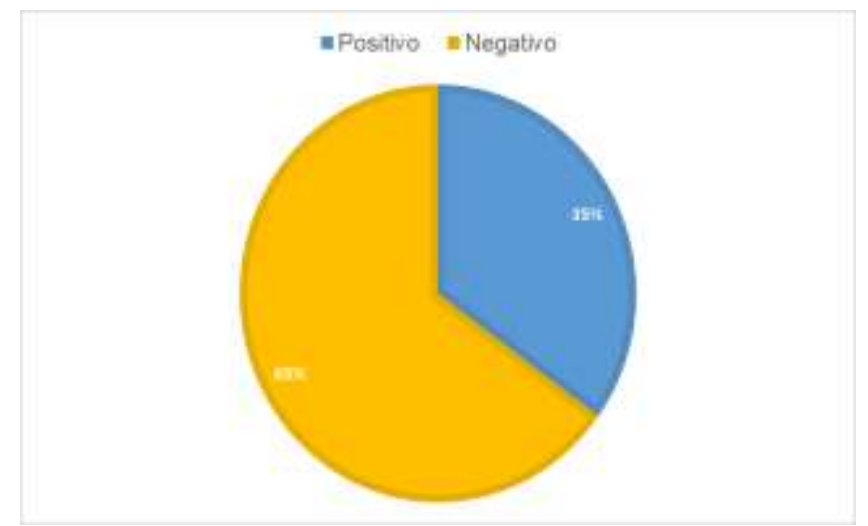




\section{Escala de Braden}

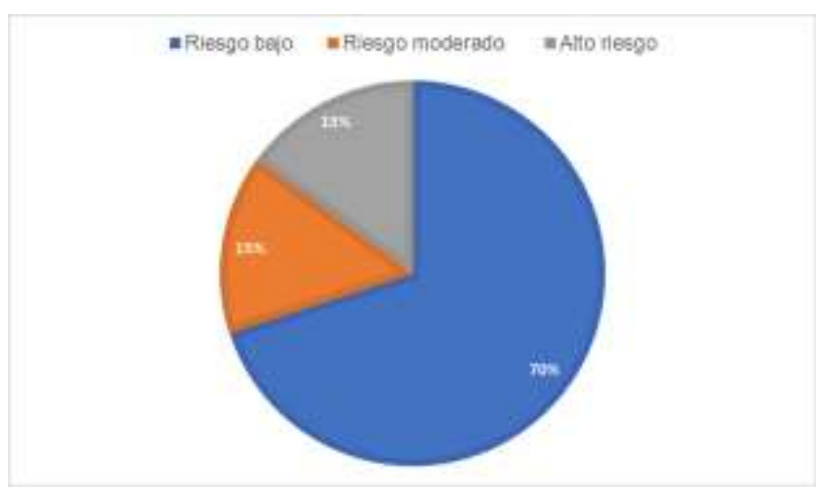

La escala de Braden se aplicó a 20 pacientes, arrojando los siguientes resultados; 14 pacientes $(70 \%)$ tienen un bajo riesgo, 3 pacientes $(15 \%)$ un riesgo moderado y 3 pacientes $(15 \%)$ un alto riesgo.

\section{Escala de Morse}

La escala de Morse se aplicó a 20 pacientes, arrojando los siguientes resultados; 5 pacientes $(25 \%)$ tienen un riesgo alto de caídas, 10 pacientes $(50 \%)$ un riesgo bajo y pacientes $(25 \%)$ sin riesgo.

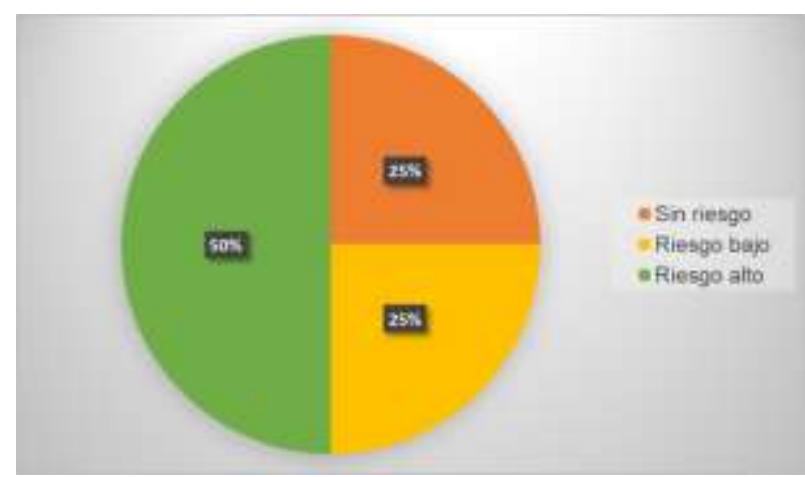

\section{CONCLUSIÓN}

Para finalizar, es vital reconocer la importancia de realizar diagnósticos de esta magnitud, ya que con ello ayudamos a mejorar la calidad de vida de los pacientes mayores, identificando a aquellos que tienen o están en riesgo de problemas nutricionales. Como futuros profesionales de la salud, debemos ampliar nuestra visión más allá de lo que aprendemos, estar en una búsqueda constante de nuevas estrategias para ayudar a las personas vulnerables, siempre optando por la prevención de aquellas enfermedades que pueden poner en alto riesgo el estado de salud de nuestros pacientes mayores, afortunadamente, a través del tiempo gracias a la ciencia, tenemos mejores herramientas de apoyo las cuales mejoran la calidad de atención que como personal de enfermería podemos otorgar, aunque esas herramientas son solo un apoyo, el trabajo duro viene de nuestra calidad de atención, con calidez humana.

Financiamiento: Este proyecto fue financiado por la empresa Semillas Hibridas de Irapuato S.A. de C.V. 
Agradecimiento a los estudiantes que participaron en la elaboración de este trabajo:

\begin{tabular}{|l|l|l||}
\hline $\begin{array}{l}\text { Aguilar Laguna María } \\
\text { Guadalupe Madeleine }\end{array}$ & Gaytán Hernández Milene & Ortiz Alfaro Daniel Alfredo \\
\hline Aguilar Morales Miriam Abigail & $\begin{array}{l}\text { Hurtado Montoya María de los } \\
\text { Ángeles }\end{array}$ & Otero Cabrera Laura Cristal \\
\hline Ávila Martínez Elizabeth Nayelli & Iturriaga Mina Karla María & Patlán Rosales Daniela Elizabeth \\
\hline Camarillo Toledo Luz Aidee & López Villegas Leslie Daniela & Ramos Cisneros Mariana \\
\hline Espinosa López Natalia Sinaí & $\begin{array}{l}\text { Lozano Torres Jesús Gabriela } \\
\text { Alejandra }\end{array}$ & Rodríguez Grana Samuel Efraín \\
\hline $\begin{array}{l}\text { Galván Bárcenas Verónica } \\
\text { Jazmín }\end{array}$ & Martínez García Diana Edith & Rodríguez Rico Iris Yunuel \\
\hline García Ramírez Andrea Michelle & $\begin{array}{l}\text { Martínez Hernández Jazmín } \\
\text { Guadalupe }\end{array}$ & Ruiz Salas Paulina Berenice \\
\hline Granados Martínez Daniela & Martínez Morales Ruth Mariela & Segura Manjarrez Ángel Guadalupe \\
\hline González Luna María Fernanda & Medrano Acosta Ana María & Vela Ramírez Jazmín Sarahí \\
\hline
\end{tabular}

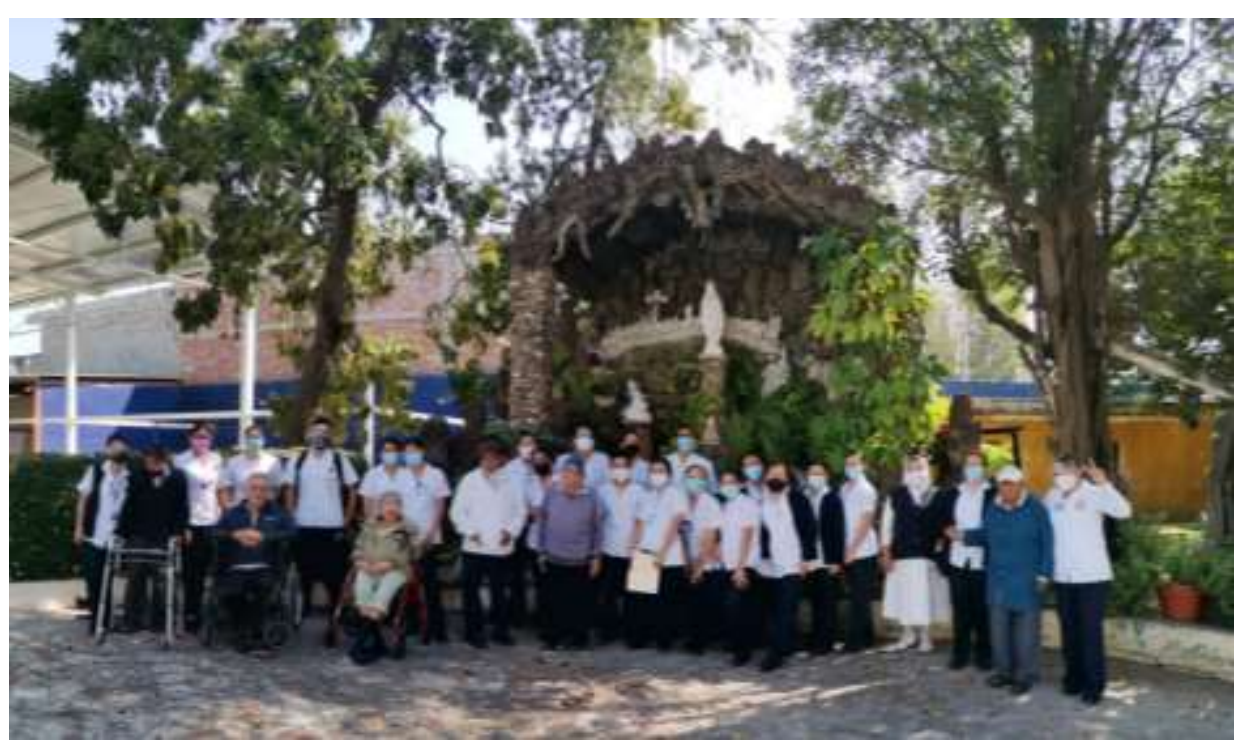




\section{REFERENCIAS BIBLIOFRAFICAS}

Calenti Millán, Gerontología y Geriatría. Valoración en intervención. Edit. Panamericana. España, 2011.

Gómez Bedoya María, (2008). El Aprendizaje en la tercera edad. Una aproximación en la clase de Ele: Los aprendientes mayores japoneses en el Instituto Cervantes de Tokio.

Instituto de la U N E S CO para la Educación, 1999. Fecha de Consulta 24 de Julio de 2021.

Hernández Triana, Manuel, 2014. Envejecimiento. Revista Cubana de Salud Pública, Fecha de consulta 23 de junio de 2016. Disponible en Disponible en: http://www.redalyc.org/articulo.oa?id=21432546011 y Práctica. Ed. Mc Graw Hill. 4ta edición. España, pp. 39-58.

Mogollón E. (2012). Una perspectiva integral del adulto mayor en el contexto de la educación. Revista Interamericana de Educación en el Adulto Mayor. 34(1), 5774. Descargado el 01 de junio de 2016 Disponible en: http://www.redalyc.org/pdf/4575/457545090005.pdf

Navarro Elías María de Guadalupe, et. Al. Cuidado de Enfermería y la Calidad de Vida en el Adulto Mayor, Universidad de Guanajuato, Universidad Nacional de Trujillo-Perú, ALTARES COSTA-AMIC Editores.

Nicola Pietro de (1985) Geriatría, Edit El Manual Moderno, México, D.F.

Novel Marti Gloria, (1991), Enfermería Psicosocial, edit. Salvat, Barcelona España, pag 5.

OMS 2015, Informe mundial del envejecimiento y la salud. Descargado el 01 de junio de 2021. ¿Disponible en http://apps.who.int/iris/bitstream/10665/186466/1/9789240694873_spa.p df?ua $=1$

Orem, E. Dorothea, Modelo de Orem Conceptos de Enfermería en la práctica, Edit. Masson- Salvat, Barcelona, 1993

Osorio Adriana y Dr. Alejandro Álvarez Mora. - Introducción a la Salud Familiar/editado por 1 ed. - San José, Costa Rica, 2004. Obtenido el día 26 de agosto de 2021.

Ramírez, Liberio Victorino; Víctor Ramírez, Ana Cecilia; (2010). Educación para adultos en el siglo XXI: análisis del modelo de educación para la vida y el trabajo en 
México ¿avances o retrocesos? Tiempo de Educar, enero-junio, 59-78. Disponible en: http://www.redalyc.org/articulo.oa?id=31116163004. Consultado el 25 de Julio de 2021.

Sociedad Española de Enfermería Geriátrica y Gerontológica, Temas de Enfermería Gerontológica, Editado por la sociedad española de geriatría y gerontología, 1999. Bergland A, Kirkevold M. Thriving in nursing homes in Norway: Contributing aspects described by residents. International Journal of Nursing Studies 2006;43(6):68191.

Burke Mary M, Walsh Mary B. 1998. Enfermería Gerontológica, Cuidados integrales del adulto mayor, Edit Elsevier Mosby, Segunda Edición, Madrid, España, pág. 602 Blumel, J., Tirado, K., \& Schiele, C. (2004). Validez de la escala de Braden para predecir úlceras por presión en población femenina. Revista Médica de Chile, 132(5), 595600. https://doi.org/10.4067/S0034-98872004000500009

Colliere MF. Encontrar el sentido original de los cuidados enfermeros. [revista en la Internet].1999; [Citado 2021 Nov 02]; 22(1): 27-31. Disponible en: https://dialnet.unirioja.es/servlet/articulo?codigo $=3558272$

Fassio Adriana, Rutty María Gabriela, Ortíz-Rojas Yenny Patricia, Aijenbon Gisela. Innovación Social, Politicas Públicas y Aprendizaje Organizacional: El Programa Nacional de Cuidados Domiciliarios [revista en la Internet]. 2015 jul [Citado 2021 Nov 02]; 7(13): 9-24. Disponible en: http://itmojs.itm.edu.co/index.php/trilogia/article/view/810/765

Frenk Julio, Gómez-Dantés Octavio. La globalización y la nueva salud pública. Salud

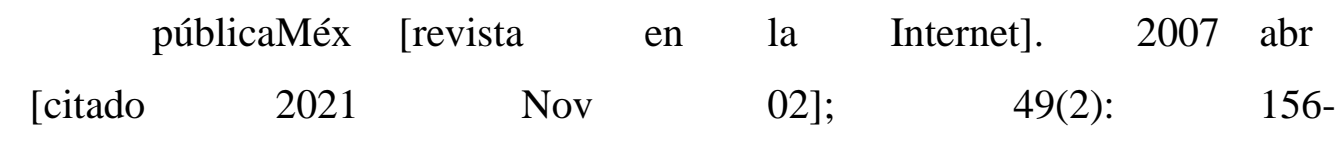

164. Disponible en:

http://www.scielo.org.mx/scielo.php?script=sci_arttext\&pid=S003636342007000200011\&lng=es.

Garcia, F., Pancorbo, P., \& Blasco, C. (2008). Escalas de valoración del riesgo de desarrollar úlceras por presión. Scielo, 19(3). https://scielo.isciii.es/scielo.php?script=sci_arttext\&pid=S1134928X2008000300005 
Gómez Bedoya María, (2008). El Aprendizaje en la tercera edad. Una aproximación en la clase de Ele: Los aprendientes mayores japoneses en el Instituto Cervantes de Tokio.

Hidalgo-Pedraza L, Blanca-Gutiérrez J, Jiménez-Díaz M d C, Grande- Gascón M L, Linares-Abad M, Relación del cuidado que demandan las personas mayores en hogares para ancianos: metaestudio cualitativo. Aquichan 201212213-227.

Disponible en: http://www.redalyc.org/articulo.oa?id=74124948002.

Fecha de consulta: 2 de noviembre de 2021.

Instituto Nacional de Geriatría. Hechos y desafíos

para un envejecimiento saludable en México. 2016. México Disponible en: http://www.geriatria.salud.gob.mx/descargas/noticias/HECHOS.pdf Consultado el 07 octubre del 2021.

Lugo-Galera Carlos, Huerta-Sobrino Cristina y Yfarraguerri-Villarreal Lucía, La Globalización Económica y su impacto en el Mercado Laboral en México, [International Journal of Good Conscience] 2014 Ags [citado Nov 2021] 9(2)6989

NéstleNutritionInstitute. (s.f.). Guía para rellenar el formulario Mini Nutritional Assessment (MNA). Obtenido de https://www.mnaelderly.com/sites/default/files/2021-10/mna-guide-spanish.pdf

Mogollón E, Una perspectiva integral del adulto mayor en el contexto de la educación. Revista Interamericana de Educación de Adultos 2012 3456-74. Disponible en: http://www.redalyc.org/articulo.oa?id=457545090005. Fecha de consulta: 2 de noviembre de 2021.

Moreno-Fergusson M E, La globalización y el conocimiento de enfermería. Aquichan 20099210-211. Disponible en: http://www.redalyc.org/articulo.oa?id=74112147001. Fecha de consulta: 2 de noviembre de 2021.

Morse, J.M.; Morse, R.M. \& Tylko, S.J.: Development of a scale to identify the fallprone patient. Canadian Journal on Aging. 1989; 8: 366-377. O'Connell, B. \& Myers, H.: The sensitivity and specificity of the Morse Fall Scale in an acute care setting. J Clin. Nurs. $2002 \quad$ Jan; 11(1): 134-6. http://www.sideso.cdmx.gob.mx/documentos/legislacion/NOM_167_SSA1.pdf 
Navarro Elías María de Guadalupe, et. Al. 2014. Cuidado de Enfermería y la Calidad de Vida en el Adulto Mayor, Universidad de Guanajuato, Universidad Nacional de Trujillo-Perú, ALTARES COSTA-AMIC Editores.

Salvà Casanovas, A. (diciembre. de 2012). El Mini Nutritional Assessment. Veinte años de desarrollo ayudando a la valoración nutricional. Revista Española de Geriatría y Gerontología., 47(6). Obtenido de https://www.elsevier.es/es-revista-revistaespanola-geriatria-gerontologia-124-articulo-el-mini-nutritional-assessmentveinte-S0211139X12002296 